Citation: Bär A, Hengartner M, Kawohl W, Konrad J, Puschner B, Clarke E, Slade M, Del Vecchio V, Sampogna G, Égerházi A, Süveges A, Krogsgaard Bording M, MunkJørgensen P, Rössler W, CEDAR study group Participation in medical decisionmaking across Europe: an international longitudinal multicenter study, European Psychiatry, in press.

\title{
Participation in medical decision-making across Europe: an international longitudinal multicenter study
}

Arlette Bär ${ }^{a *}$, Michael P. Hengartner ${ }^{b *}$, Wolfram Kawohl ${ }^{a}$, Jana Konrad ${ }^{c}$, Bernd Puschner ${ }^{c}$,

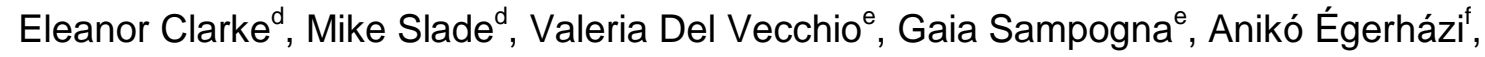
Ágnes Süveges ${ }^{\dagger}$, Malene Krogsgaard Bording ${ }^{9}$, Povl Munk-Jørgensen ${ }^{\mathrm{h}}$, Wulf Rössler ${ }^{\mathrm{a}}$, and the CEDAR study group**

${ }^{a}$ Department of Psychiatry, Psychotherapy and Psychosomatics, Psychiatric Hospital, University of Zurich, PO Box 1930, 8021 Zurich, Switzerland

${ }^{\mathrm{b}}$ Department of Applied Psychology, Zurich University of Applied Sciences (ZHAW), Pfingstweidstrasse 96, PO Box 707, 8037 Zurich, Switzerland

${ }^{c}$ Section Process-Outcome Research, Department of Psychiatry II, Ulm University, LudwigHeilmeyer-Str. 2, 89312 Günzburg, Germany

${ }^{d}$ Kings College London, Section for Recovery, Institute of Psychiatry, Psychology \& Neuroscience, 16 De Crespigny Park, London SE5 8AF, UK

${ }^{e}$ Department of Psychiatry, University of Naples SUN, Largo Madonna delle Grazie 1, 80138 Naples, Italy 
${ }^{f}$ Department of Psychiatry, Medical and Health Science Centre, University of Debrecen, 98, Nagyerdei krt. H-4012, Debrecen, Hungary

${ }^{9}$ Aalborg University Hospital, Psychiatry, Mølleparkvej 10, Aalborg, 9000, Denmark

${ }^{\mathrm{h}}$ Department M, Aarhus University Hospital, Skovagervej 2, 8240 Risskov, Denmark

${ }^{*}$ Both authors contributed equally 
Corresponding author:

Arlette Bär Deucher

Department of Psychiatry, Psychotherapy and Psychosomatics

Psychiatric Hospital, University of Zurich

PO Box 1930

8021 Zurich

SWITZERLAND

Telf.: +4144744 4785

Fax: +41447444788

Email address: arlette.baer@puk.zh.ch

${ }^{* *}$ CEDAR Study Group: Ulm (Bernd Puschner [chief investigator], Katrin Arnold, Esra Ay, Thomas Becker, Jana Konrad, Sabine Loos, Petra Neumann, Nadja Zentner); London (Mike Slade, Eleanor Clarke, Harriet Jordan); Naples (Mario Maj, Andrea Fiorillo, Corrado De Rosa, Valeria Del Vecchio, Domenico Giacco, Mario Luciano, Gaia Sampogna, Heide Gret Del Vecchio, Pasquale Cozzolino, Antonio Salzano); Debrecen (Anikó Égerházi, Tibor Ivánka, Marietta Nagy, Roland Berencz, Theodóra Glaub, Ágnes Süveges, Attila Kovács, Erzsébet Magyar); Aalborg (Povl Munk-Jørgensen, Malene Krogsgaard Bording, Helle Østermark Sørensen, Jens-Ivar Larsen); Zurich (Wolfram Kawohl, Wulf Rössler, Arlette Bär, Susanne Krömer, Jochen Mutschler, Caitriona Obermann). 


\section{ABSTRACT}

Background: The purpose of this paper was to examine national differences in the desire to participate in decision-making of people with severe mental illness in six European countries. Methods: The data was taken from a European longitudinal observational study (CEDAR; ISRCTN75841675). A sample of 514 patients with severe mental illness from the study centers in Ulm, Germany, London, England, Naples, Italy, Debrecen, Hungary, Aalborg, Denmark and Zurich, Switzerland were assessed as to desire to participate in medical decision-making. Associations between desire for participation in decision-making and center location were analyzed with generalized estimating equations.

Results: We found large cross-national differences in patients' desire to participate in decision-making, with the center explaining $40 \%$ of total variance in the desire for participation $(p<0.001)$. Averaged over time and independent of patient characteristics, London (mean=2.27), Ulm (mean=2.13) and Zurich (mean=2.14) showed significantly higher scores in desire for participation, followed by Aalborg (mean=1.97), where scores were in turn significantly higher than in Debrecen (mean=1.56). The lowest scores were reported in Naples (mean=1.14). Over time, desire for participation in decision-making increased significantly in Zurich $(b=0.23)$ and decreased in Naples $(b=-0.14)$. In all other centers, values remained stable.

Conclusions: This study demonstrates that patients' desire for participation in decisionmaking varies by location. We suggest that more research attention be focused on identifying specific cultural and social factors in each country to further explain observed differences across Europe.

Keywords: Clinical decision-making, Patient participation, Multicenter study, Severe mental illness 


\section{Introduction}

\section{Patients' Autonomy}

One of the first empirical studies on patient participation in medical decision-making examined whether patients wish to make decisions themselves [1]. Autonomy refers to the decision-making dimension of the patient's role, and enhancing patient autonomy means helping patients to make their own decisions [2]. Considerations of a patient's autonomy in the health care context tend to focus most strongly on the idea that patients should be allowed and enabled to make autonomous decisions about their health care [3-5]. Patient involvement and patient participation in decision-making are therefore regarded as a feature of good quality of care, which is valued in its own right $[6,7]$. Nowadays, autonomy is a key concern in biomedical ethics and many countries have passed legislation to protect patients' autonomy and to provide informed consent for medical interventions [3].

\section{Shared Decision-Making}

Shared decision-making means that information exchange is a two-way process during consultation. Both the health care professional and the patient engage in deliberation and take the decision. Self-determination of patients and their right to a comprehensive explanation of the illness and all its treatment possibilities are the fundamental prerequisites of this model [8]. The model contrasts with (i) the 'paternalistic' model, where information is given to the patient and the deliberation and decision are made by the health care professional, or (ii) an 'informed' model, where information is given to the patient and the patient deliberates and decides [9]. The NICE (National Institute for Clinical Excellence) has continuously promoted shared decision-making by ensuring that its clinical guidelines address issues of importance to both patients and health care professionals [10]. Shared decision-making is recommended in systematic reviews [11].

\section{Preferences for participation in decision-making among patients with serious mental illness}


Reviews of shared decision-making in persons with schizophrenia [8] and depression [12] showed that patients and physicians found shared decision-making acceptable and did in fact engage in shared decision-making, which resulted in improvements in patients' knowledge about their illness and a higher level of perceived involvement in decision-making. Patients generally express a desire for greater participation in decision-making about psychiatric care than what they are currently experiencing. Concerning the use of new psychiatric medication, most participants prefer autonomous or shared roles. A passive role in medication decisions in psychiatric care was observed to be less likely than a passive role in general medical care [13].

Hamann et al. [8] showed that the desire to participate in decision-making was higher in inpatients with experiences of involuntary treatment, with negative attitudes toward medication, with a higher level of education, with lower treatment satisfaction (e.g. general treatment satisfaction, trust in clinicians, and fairness), with better-perceived decision-making skills, in patients of female gender and in younger patients., It was, furthermore, demonstrated that patients with schizophrenia strongly wish to participate in medical decisions on an equal footing with their doctors, rather than to completely take over decisional control $[8,14,15]$. Moreover, a large study in the United States with a focus on outcomes in adults diagnosed with depressive disorders showed that greater involvement in depression care resulted in higher participant satisfaction and lower depression scores [16]. In the CEDAR study people with severe mental illness stated a preference for a shared decision-making style [17]. Patients indicated that involvement in decision-making during their last treatment session was mainly shared (shared decision model) [17] and most people wanted to receive information on all aspects of their treatment [18].

\section{Influence of Culture}

There is little knowledge about what actually constitutes 'cultural' differences in mental health. Triggered by socio-political factors (among others), participation preferences are considered to have increased in the past decades $[19,20]$. Movements promoting the 
rights of citizens, consumers and patients, who in western countries have become increasingly active since the 1960s, are considered to have changed the 'culture' of the medical health care system [21, 22]. Overall, 'culture' is reported to be an important factor explaining differences in patients' preferences, but results remain inconsistent [15]. The extent to which patients expect to be actively involved in treatment decisions varies according to the prevailing medical culture. If it is paternalistic, both doctors and patients are likely to assume that decisions are the responsibility of the doctor only, whereas in a more egalitarian culture a partnership or shared decision-making approach may be preferred [23].

In a conceptual paper, Charles et al. [24] discussed the influence of culture on the process of treatment decision-making and, in particular, shared treatment decision-making in the physician-patient encounter. It was noted that the nature of the encounter is influenced by cultural expectations, e.g. the patients' and physicians' role expectations and expectations as to who is involved in decision-making. Finally, in the DIALOG trial (a computer-mediated procedure to structure patient-clinician dialogue) the effectiveness of a novel intervention was studied across six European community mental healthcare settings (incl. Germany, UK, Switzerland, Spain). It was assessed whether the effectiveness of structured patient-clinician communication varies between services in different countries. In the centers in which the DIALOG intervention proved most effective (namely London and Granada), patients had comparatively high symptom levels (London), more unmet needs for care, a shorter history in mental healthcare and fewer hospital admissions (London and Granada) [25].

Although patients' involvement and participation in decision-making is regarded as a feature of good quality of care [3], little is known about national differences in the preferences for participation in decision-making of people with severe mental illness. The main objective of the present study was thus to study whether patients' participation preferences differed across six European countries, both in addition to and independent of patient characteristics. Since patients' preferences are likely to develop over time and may change at different stages of their illness $[17,26]$, we also examined whether there was an interaction effect 
between study center and time. Based on the literature we hypothesized that the desire for participation in decision-making would vary substantially across countries.

\section{Material and methods}

\section{Sample}

The data were taken from the study "Clinical Decision-making and Outcome in Routine Care for People with Severe Mental Illness“ (CEDAR; ISRCTN75841675) [17]. CEDAR is a naturalistic prospective longitudinal observational study with bimonthly assessments during a 12-month observation period (T0-T6) carried out in six European countries (Germany, UK, Italy, Hungary, Denmark and Switzerland). First, CEDAR established a methodology to assess clinical decision-making in people with severe mental illness. Specific instruments were developed to measure clinical decision-making style, key elements of clinical decision-making in routine care, as well as clinical decision-making involvement and satisfaction from patient and clinician perspectives. Second, these instruments were applied in a multi-national prospective observational study. This study investigated the immediate, short- and long-term effect of clinical decision-making on crucial dimensions of clinical outcome (symptom level, quality of life, needs) by taking into account significant variables moderating the relationship between clinical decision-making and outcome. Participants in the CEDAR study were recruited between November 2009 and December 2010 from caseloads of outpatient/community mental health services at six centers: Department of Psychiatry II, Ulm University, Germany (coordinating center); South London and Maudsley NHS Foundation Trust, London, the U.K.; the Department of Psychiatry at University of Naples SUN, Italy; the Department of Psychiatry at Debrecen University, Hungary; the Unit for Psychiatric Research at Aalborg University Hospital (former Aarhus University Hospital), Denmark; and the former Department of General and Social Psychiatry at the University of Zurich, Switzerland. Ethics committees approved the study in all centers. After being informed about the goal, the funding of the study, the right to refuse to participate or to withdraw consent without adverse consequences, all participants gave their 
written informed consent. The CEDAR study was conducted in accordance with the Helsinki Declaration of 1975, as revised in 2008, of the World Medical Association [17].

The six study sites reflect the diversity across Europe in the organization of mental health services. Ulm, Germany (coordinating center): The department is responsible for the provision of mental health care in a large catchment area in rural Bavaria (population 671 000). Multidisciplinary teams (psychiatrists, psychologists, social workers, nurses and occupational therapists) offer a full range of pharmacological and psychosocial interventions in inpatient, outpatient and daycare clinics. The department collaborates closely with officebased psychiatrists and psychotherapists in the area. London, UK: The site comprises three specialist community teams: early psychosis, assertive outreach and rehabilitation and recovery. All teams are multidisciplinary, comprising clinical psychology, nursing, occupational therapy, psychiatry and social work professionals, as well as support workers and administrative staff. These teams provide services across the London Borough of Croydon (population 330000 ) as part of a range of services for adults aged 18-65, including three community mental health teams, a home treatment team, a community forensic team and in-patient beds. Naples, Italy: The department includes inpatient and outpatient units and a 1-day hospital. The outpatient units include specialist clinical teams for the management and treatment of psychotic disorders, mood disorders, eating disorders and obsessivecompulsive disorders. Specialist teams for early detection and management of psychoses and for cognitive and psychosocial rehabilitation are available. Debrecen, Hungary: The department provides in- and outpatient mental health care for the city of Debrecen (population 200 000). The team is completed by an occupational therapist and a social worker professional that maintain contact with the regional rehabilitation institutions and mental homes. Aalborg, Denmark: The Psychiatry Region North includes various treatment centers, including inpatient treatment, outpatient teams and early psychosis teams. The collaborating centers in the CEDAR study were organized within the universities of Aarhus, Aalborg, Copenhagen and Southern Denmark. Others were provincial hospitals with links to Aarhus University. Furthermore, CEDAR collaborated with office-based psychiatrists. Zurich, 
Switzerland: The department takes responsibility for a defined catchment area in Zurich City of about 390000 inhabitants. It comprises 488 beds and additionally offers specialized care in a crisis center and a center for psychiatric rehabilitation [17].

A convenience sample of patients was recruited from mental teams. Potential patient participants were identified by clinicians. The referring clinician asked patients who met inclusion criteria to consent to researcher contact. A researcher then met the patient, explained the study and obtained signed informed consent. In nearly all study centers the patient nominated a key clinician of any profession whom they saw regularly. In Denmark, it was reversed: patients were recruited via staff. Data was collected using questionnaires (filled in by the patient and his or her key worker) or through interviews conducted by the CEDAR researchers. Data entry was via computer or paper-pencil forms. See Puschner et al. [27] for further details on the rationale and design of the CEDAR study.

Participating staff from the CEDAR study were in their mid-40s on average and their mean time of working in mental health services was 15 years. The category of professions included psychiatrists, psychologists, social workers and others (nurse, district nurse, support time and recovery worker and psychiatric trainee) [27].

\section{Participants}

Inclusion criteria were: adult age (18-60 years) at intake, mental disorder of any kind as main diagnosis established by case notes or staff communication using SCID criteria [28], presence of severe mental illness (Threshold Assessment Grid (TAG) $\geq 5$ points and illness duration $\geq 2$ years; [29]); expected contact with mental health services (excluding inpatient services) during the time of study participation; sufficient command of the host country's language; and capability of giving informed consent. Exclusion criteria were: main diagnosis of learning disability, dementia, substance use or organic brain disorder; cognitive impairment severe enough to make it impossible to give meaningful information on study measures; and treatment by forensic mental health services [17].

A total of 708 patients were screened for eligibility, of which 588 were included. The 
reason for exclusion was not meeting inclusion criteria $(n=120)$. The study uses data of participants $(n=514)$ who completed the time points baseline (T0) and one-year follow-up (T6). All data on patients' socio-demographic and clinical characteristics were assessed through the Client Sociodemographic and Service Receipt Inventory - European Version (CSSRI-EU) questionnaire at baseline (T0) and at one-year follow-up (T6) [17].

\section{Measures and Instruments}

The Clinical Decision-Making Style (CDMS) [30] is an adaptation of the Autonomy Preference Index (API; [1]) for use in people with severe mental illness. The subscale "Participation in Decision-Making" (CDMS-PD) consists of 15 items, each rated on a fivepoint Likert scale ranging from 0-4 (with a higher score indicating a higher desire by the patient to be an active participant in decision-making). Categorical sum scores were formulated on the basis of utility, where an emphasis was placed on separating categories according to clinical meaningfulness. Categories of decision-making models according to the CDMS-PD subscale were "passive" (CDMS-PD $<1.5$, defined as the clinician taking the decision, with or without considering the views of the patient), "shared" (1.5-<=CDMS$\mathrm{PD}<=2.5$, defined as decisions being made jointly between clinician and patient) and "active" (CDMS-PD>2.5, defined as the patient taking the decision, with or without considering the views of the clinician). These categories distinguish groups by their ordinal nature but not by a specific value assigned to each category. The paternalistic model corresponds to a passive involvement of the patient; the shared decision-making and the informed choice model correspond to an active involvement of the patient. The CDMS was measured at baseline (T0) and at one-year-follow-up (T6). For a detailed account of the CDMS, see Puschner et al. [30].

\section{Statistical Analysis}

Baseline characteristics across centers were examined with contingency tables and $\chi^{2}$ significance tests for categorical variables and with independent sample t-tests for 
continuous variables. The repeated measures of participation in medical decision-making over time were examined with a series of generalized estimating equations (GEE). These models were introduced to fit regression analyses that account for within-subject correlation, which is an inherent part of longitudinal studies that rely on repeated measures [31]. Owing to the distribution of the dependent variable (CDMS-PD), a normal distribution with an identity link-function best fitted our data. A robust maximum likelihood estimator was used to reduce the effects of outliers and influential observations. The intercept and slope factor was included in all analyses, which is a common procedure in longitudinal data modeling [32]. To adjust for the within-subject correlation, the slope factor was also modeled as an interaction effect with the predictor variable to examine changes in the desire to participate in decisionmaking over time. To examine group differences, pairwise comparisons with sequential Bonferroni correction for multiple testing were conducted. Interaction effects of categorical and continuous predictors with the time-trend were indicated with unstandardized regression coefficients (b). The proportion of total variance explained in the repeated outcome was estimated with a pseudo- $\mathrm{R}^{2}$, which is obtained by dividing the variance of the full model (i.e., dispersion parameter) by the variance of the repeated dependent variable. All analyses were carried out with SPSS version 20 for Macintosh.

\section{Results}

- Table 1-

The descriptive statistics are indicated in Table 1. For all variables the differences across centers were statistically significant. According to a series of bivariate GEE comprising center and the nine patient variables indicated in Table 1, center was the substantially strongest bivariate predictor of participation in decision-making. Center explained $39.9 \%$ of total variance in the CDMS-PD over time $\left(\chi^{2}=529.065, \mathrm{df}=5, \mathrm{p}<0.001\right)$, which corresponds to a large effect size. The highest mean CDMS-PD scores pooled over 
time were reported in London (2.28), Ulm (2.14) and Zurich (2.15), followed by Aalborg (1.98), which in turn was significantly higher than Debrecen (1.53). The distinctly and statistically significantly lowest CDMS-PD score was reported in Naples (1.12). In Zurich, the CDMS-PD increased significantly from to to t6, whereas in Naples it declined significantly over time; at the other study sites it remained stable (see Table 2).

-Table 2-

Since patients' characteristics between the respective centers differed significantly (see Table 1), possible cultural differences on CDMS could be attributed to regional differences as well as to distinct patient characteristics. Therefore, to answer the question of whether cultural influences were independent of distinct patient characteristics across centers, we regressed all nine predictors from Table 1 (i.e., gender, age, marital status, ethnic group, years in school, living situation, employment status, state benefits and diagnosis) separately on the CDMS-PD. Of the nine predictor variables listed in Table 1, age $\left(\chi^{2}=10.967, d f=1, p=0.001\right)$, marital status $\left(\chi^{2}=14.133, d f=1, p<0.001\right)$, living situation $\left(\chi^{2}=36.674, d f=2, p<0.001\right)$ and state benefits $\left(\chi^{2}=6.628, d f=1, p=0.010\right)$ were significantly related to the repeated measures of the CDMS-PD according to bivariate GEE. These variables were thus included in addition to center in a fully adjusted GEE model. We additionally added the repeated TAG score, which is a measure of illness severity and which also related weakly to the CDMS-PD $\left(\chi^{2}=5.954, \mathrm{df}=1, \mathrm{p}=0.015\right)$. In this multivariate model, only center $\left(\chi^{2}=184.221, \mathrm{df}=5, \mathrm{p}<0.001\right)$ and the interaction term between center and time $\left(\chi^{2}=33.244, \mathrm{df}=6, \mathrm{p}<0.001\right)$ remained statistically significant predictors of the CDMS-PD. The results for center are shown in Table 3. The adjustment for significant covariates did not alter the results substantially. In accordance with the unadjusted analysis, London (2.27), UIm (2.15) and Zurich (2.14) showed the highest pooled CDMS scores over time, followed by Aalborg (1.96), Debrecen (1.54), and Naples (1.14). In this multivariate model, we again observed a significant increase in the CDMS-PD over time in Zurich as well as a significant 
decrease over time in Naples.

-Table 3-

\section{Discussion}

The aim of this study was to examine differences between European countries in patients' participation in medical decision-making by focusing on psychiatric services in six different European countries. We found large differences between the study centers in patients' wishes to participate in decision-making, with the center location as the predictor variable explaining $40 \%$ of total variance in the CDMS-PD. Moreover, those national differences were independent of patient characteristics including gender, age, marital status, ethnic group, years in school, living situation, employment status, state benefits and diagnosis

Participants from North-western Europe (Ulm, London, Aalborg and Zurich) showed moderate scores over time on CDMS-PD, indicating a preference for shared decisionmaking, with a higher preference for active decision-making mainly in UIm, London and Zurich. On the other hand, participants from Southern Europe (Naples) showed a preference for passive decision-making. While participants from Zurich increased in their wish to participate in decision-making over time, the opposite was observed in Naples, where participants' initial low preference for autonomous decision-making declined further after twelve months.

Our results are in line with the results of a study on communication, information, involvement and choice in clinical decision-making, in which 1000 interviews were carried out in nine countries (e.g. UK, Germany, Poland, Italy) with random samples of the adult population. The results show that a more paternalistic view of the doctor-patient relationship prevailed in Poland and Spain compared to the six other countries: $91 \%$ of Swiss and $87 \%$ of German respondents believed that the patient should have a key role in treatment decisions, either by sharing responsibility with the doctor or by being the primary decision-maker. The proportion 
of Polish patients who shared that opinion was only 59\%, and in Spain it was only $44 \%$ [33]. Similar results were obtained in a study with patients with urinary incontinence. In this study, preferences for involvement in treatment decision-making in 15 European countries were explored. Women in North-western European countries (e.g. Germany, UK and Switzerland) wished to be involved in the treatment decision-making process with a collaborative role. Women in Denmark opted for a more active role, preferring more active involvement than women in Southern European countries (e.g. Greece, Portugal, Spain), where passive involvement was clearly favored. The results suggest that particularly in Southern European countries many women prefer a passive role in treatment decision-making [34]. Cultural barriers - for example, patients' and health professionals' beliefs about their roles within the consultation - may be hard to overcome in some countries. It is therefore important that professionals are prepared to support patients in this process. For instance, patients are unlikely to become involved in self-management education programmes if they do not believe they have a key part to play in managing their health. Social inequalities may also inhibit changes in patient involvement. Those with lower levels of education and lower incomes may feel less confident about becoming involved in their care and in evaluating health information. They are also less likely to have access to computers and the Internet. The digital divide is more apparent in some European countries than in others. A survey of citizen's use of e-health services found that health-related use of the Internet was most frequent in northern European countries, such as Denmark (62\%), Norway (59\%) and Germany $(49 \%)[35,36]$. Eastern European countries such as Poland and Latvia reported a $42 \%$ and $35 \%$ health-related use of the Internet, respectively, while southern Europe had the lowest proportion of Internet health users, with 30\% in Portugal and 23\% in Greece [35]. A recent study found that Hispanics preferred a more passive role in mental health decisionmaking while non-Hispanics preferred a more active role. The authors discussed several reasons for these findings. Some of them could be applied to our findings. First, patients' health worldview, including health belief models, treatment preferences, involvement of family, greater trust in their physician and attitude may vary and influence a patient's 
preference for participation (influence by cultural attitudes and tradition) [37-40]. Second, perceived lack of knowledge, lack of self-efficacy or a learned response influenced by cultural attitudes may contribute to this as well [38-40]. Further potential reasons identified were the distinct organizational models in operation in the six European countries, which may lead to different ways of thinking about participation in medical decision-making, competence, vulnerability and risk to self, which raise particularly difficult issues in psychiatry [41-43].

In some countries (e.g. Switzerland), patients can immediately visit a specialist clinician if they wish so, while in others (e.g. Italy, Denmark and England), patients must first secure a referral from a GP. The right to choose the timing and location of hospital treatment is well established in some countries (e.g. Germany), whereas long waits and limited choice of location are standard in others (e.g. UK). Policy makers in the UK and elsewhere are attempting to speed up access by making it easier for patients to travel to hospitals with spare capacity if they wish to [23]. Another reason could be that some patients' wish for passivity may be genuine and may reflect their wish to be taken care of or to be free of responsibility for treatment failure [44]. A further reason is the influence of the physician's expectations of patient role. There is evidence of differences between doctors in eastern and western Germany concerning the nature of 'right' treatment and the 'right' patient role. In summary, physicians in the east have more paternalistic attitudes toward patients. This data is mirrored by a telephone survey showing that patients from Poland view their doctors as more paternalistic than patients in most western European countries. Thus, patients (in eastern Germany) who have experienced more paternalistic doctors already expect a more passive role $[14,15]$. In general, culture has so far been mostly neglected in the medical literature on treatment decision-making [24] and we can only comment on its possible influence on role preferences. Further research would be required to identify specific cultural and social factors in each country that might explain the observed cross-national differences.

\section{Limitations}


There are some limitations that have to be addressed. The primary limitation was that sample characteristics were heterogeneous across centers, which could decrease comparability and generalizability of our findings. A further limitation was that the CDMS was only measured twice instead of several times and that the CEDAR study lasted only one year. Having more measurement occasions would be useful to develop a better understanding of how patients' wish for participation might change over time and to better understand the complexity of medical decision-making in mental health services.

Furthermore, only six different countries were examined. Additional countries and different locations within the same country would enhance both international comparability and interpretation of the results. Furthermore, our study focused only on "culture" and "patient characteristics" as factors that influence patients' preferences for involvement in decisionmaking. Other factors (e.g. the amount of knowledge patients have acquired about their condition) that influence patients' preference for involvement in decision-making were not considered in detail in this study. Another limitation is the patients' self-reported preferences and the failure to consider the impact of professional preferences on the results. In this paper only patient perspectives were considered. It is well established that attitudes do not immediately relate to behavior. Thus, for example, a patient who expresses high participation preferences in the questionnaire might at the same time behave entirely passively during a consultation because his doctor behaves very authoritatively. There is also evidence that patients - to varying extents - learn to present themselves and their illness according to cues from professionals. This learning process may also in part explain the changes over time in preferences observed. Finally, no data was collected about the cultural and social aspects of the professionals involved and the study sites are very distinct from one another. For further studies on this topic, the factors mentioned should be taken into account.

\section{Conclusions}

The data shows that center location has an impact on the patients' desire for participation in decision-making. The influence of location has so far been a relatively 
neglected topic in the medical literature on treatment decision-making. Cultural influences can be a major factor affecting the dynamics of the physician-patient interaction, the type of decision-making process used, the potential for reaching a mutually agreed upon decision, and the satisfaction of both parties with both the process and the treatment decision. More research in these areas is certainly needed [22]. Involving patients in decision-making is one of many ways through which quality of health care could be improved [45]. Preference for participation in medical decision-making varies widely. Involvement may not be acceptable or appropriate for everyone $[17,18]$. Patients should be empowered so that they experience the benefits associated with involvement. Among other things, empowerment (i.e. within mental health services, including becoming an empowered member of society) is one of the recovery processes that have the most proximal relevance to clinical research and practice [46]. The current conceptualizations of recovery are primarily based on Western European and North American models [47]. Empowerment may therefore relate to a particular cultural value, which we found to be more present in Northern and Western Europe than in Central and Southern Europe. This highlights the culture-specific values inherent in evidence-based medicine, and the need to ensure that treatment approaches - especially in relation to interpersonal processes - are consistent with local cultural values. 


\section{Acknowledgements}

The CEDAR study is funded by a grant from the Seventh Framework Programme (Research Area HEALTH-2007-3.1-4 Improving clinical decision-making) of the European Union (Grant no. 223290). CEDAR is a multi-center collaboration between the Section Process-Outcome Research, Department of Psychiatry II, Ulm University, Germany; the Section for Recovery, Institute of Psychiatry, King's College London, U.K.; the Department of Psychiatry, University of Naples SUN, Italy; the Unit for Psychiatric Research, Aalborg Psychiatric Hospital, Aarhus University Hospital, Denmark; the Medical and Health Science Center, Department of Psychiatry, University of Debrecen, Hungary; and the Department of General and Social Psychiatry, University of Zurich, Switzerland.

CEDAR Study Group: Ulm (Bernd Puschner (chief investigator), Thomas Becker, Katrin Arnold, Esra Ay, Jana Konrad, Sabine Loos, Petra Neumann, Nadja Zentner); London (Mike Slade, Elly Clarke, Harriet Jordan); Naples (Mario Maj, Andrea Fiorillo, Valeria Del Vecchio, Corrado De Rosa, Domenico Giacco, Mario Luciano, Gaia Sampogna, Lucia Del Gaudio, Pasquale Cozzolino, Heide Gret Del Vecchio, Antonio Salzano); Debrecen (Anikó Égerházi, Tibor Ivánka, Marietta Nagy, Roland Berencz, Teodóra Glaub, Ágnes Süveges, Attila Kovacs, Erzsebet Magyar); Aalborg (Povl Munk-Jørgensen, Malene Krogsgaard Bording, Helle Østermark Sørensen, Jens-Ivar Larsen); Zurich (Wolfram Kawohl, Wulf Rössler, Arlette Bär, Susanne Krömer, Jochen Mutschler, Caitriona Obermann).

\section{Competing interests}

The author(s) declare that they have no competing interests. 


\section{References}

[1] Ende J, Kazis L, Ash A, Moskowitz MA. Measuring patients' desire for autonomy: decision making and information-seeking preferences among medical patients. J Intern Med 1989; 4: 23-30.

[2] Nease RF Jr, Brooks WB. Patient desire for information and decision-making in health care decisions: the Autonomy Preference Index and the Health Opinion Survey. J Intern Med 1995; 10: 593-600.

[3] Beauchamp TL, Childress JF. Principles of biomedical ethics (6th ed.). Oxford University Press; 2009.

[4] Gillet G. Autonomy and selfishness. Lancet 2008; 372: 1214-1215.

[5] Walker RL. Medical ethics needs a new view of autonomy. J Med Philos 2009; 33: 594608.

[6] Entwistle VA, Watt, IS. Patient involvement in treatment decision-making: The case for a broader conceptual framework. Patient Educ Couns 2006; 63: 268-278.

[7] Farrel C. Patient and public involvement in health: the evidence for policy implementation in London. Department of Health 2004; Product number 40119, Getway reference 2880.

[8] Hamann J, Cohen R, Leucht S, Busch R, Kissling W. Do patients with schizophrenia wish to be involved in decisions about their medical treatment? Am J Psychiatry 2005; 162: 23822384

[9] Charles C, Gafni A, Whelan T, O’Brien MA. Decision-making in the physician-patient encounter: revisiting the shared treatment decision-making model. Soc Sci Med 1999; 49: $651-661$.

[10] Stokes T. NICE Clinical Guidelines: involving patients, sharing decision-making, considering cost effectiveness. Huisarts Wet 2010; 53: 20-21.

[11] Duncan E, Best C, Hagen S. Shared decision making interventions for people with mental health conditions (Protocol). Cochrane Syst Rev. 2010 Jan 20;(1):CD007297. doi: 10.1002/14651858.CD007297.pub2. 
[12] Clever SL, Ford DE, Rubenstein LV, Rost KM, Meredith LS, Sherbourne CD, Wang NY, Arbelaez JJ, Cooper LA. Primary care patients' involvement in decision-making is associated with improvement in depression. Med Care 2006; 44: 398-405.

[13] Adams JR, Darke RE, Wolford GL. Shared decision-making preferences of people with severe mental illness. Psychiatr Serv. 2007; 58: 1219-1221.

[14] Hamann J, Mendel R, Reiter S, Cohen R, Bühner M, Schebitz M, Diplich S, Kissling W, Berthele A. Why Do Some Patients With Schizophrenia Want to Be Engaged in Medical Decision Making and Others Do Not? J Clin Psychiatry 2011; 72: 1636-1643.

[15] Hamann J, Bieber Ch, Elwyn G, Wartner E, Hörlein E, Kissling W. Toegel Ch, Berth H, Linde K, Schneider A. How do patients from eastern and western Germany compare with regard to their preferences for shared decision making? European Journal of Public Health $2011 ; 22: 469-473$.

[16] Swanson KA, Bastani R, Rubenstein LV, Meredith LS, Ford DE. Effect of mental health care and shared decision making on patient satisfaction in a community sample of patients with depression. Med Care Res Rev. 2007; 64: 416-30.

[17] Puschner B, Becker T, Mayer B, Jordan H, Maj M, Fiorillo A, Égerházi A, Ivánka T, Munk-Jørgensen P, Krogsgaard Bording M, Rössler W, Kawohl W, Slade M; CEDAR study group. Clinical decision making and outcome in the routine care of people with severe mental illness across Europe (CEDAR). Epidemiol Psychiatr Sci. 2016; 25:69-79. doi: 10.1017/S204579601400078X.

[18] Giacco D, Luciano M, Del Vecchio V, Sampogna G, Slade M, Clarke E, Nagy M, Egerhazi A, Munk-Jørgensen P, Bording MF, Kawohl W, Rössler W, Zentner N, Puschner B, Fiorillo A; CEDAR study group. Desire for information of people with severe mental illness. Soc Psychiatry Psychiatr Epidemiol. 2014; 49: 1883-1891. doi: 10.1007/s00127-014-0901-5. [19] Chewning B, Bylund CL, Shah B, et al. Patient preferences for shared decisions: A systematic review. Patient Educ Couns 2012; 86: 9-18.

[20] Say R, Murtagh M, Thomson R. Patients' preference for involvement in medical decision making: a narrative review. Patient Educ Couns 2006; 60: 102-114. 
[21] Coulter A. Paternalism or partnership? Patients have grown up-and there's no going back. BMJ 1999; 319: 719-720.

[22] Gillespie R, Florin D, Gilliam S. Changing Relationships: Findings from the Patient Involvement Project. London: King's Fund, 2002.

[23] Coulter A, Jenkinson C. European patients' views on the responsiveness of health systems and healthcare providers. European Journal of Public Health 2005; 15: 355-360. [24] Charles C, Gafni A, Whelan T, O'Brien MA. Cultural influences on the physician-patient encounter. The case of shared treatment decision-making. Patient Educ Couns 2006; 63: 262-267.

[25] van den Brink R, Wiersma D, Wolters K, Bullenkamp J, Hansson L, Lauber C, MartinezLeal R, McCabe R, Rössler W, Salize H, Svensson B, Torres-Gonzales F, Priebe S. Nonuniform effectiveness of structured patient-clinician communication in community mental healthcare: an international comparison. Soc Psychiatry Psychiatr Epidemiol. 2011; 46: 685693. doi: 10.1007/s00127-010-0235-x.

[26] Butow PN, Maclean M, Dunn SM, Tattersall MH, Boyer MJ. The dynamics of change: cancer patients' preferences for information, involvement and support. Ann Oncol 1997; 8: 857-863.

[27] Puschner B, Steffen S, Slade M, Kaliniecka H, Maj M, Fiorillo A, Munk-Jorgensen P, Larsen JI, Egerhazi A, Nemes Z, Rössler W, Kawohl W, Becker T. Clinical decision making and outcome in routine care for people with severe mental illness (CEDAR): study protocol. BMC Psychiatry 2010; 10: 90 doi:10.1186/1471-244X-10-90.

[28] First MB, Spitzer RL, Gibbon M, Williams JBW. Structured Clinical Interviews for DSM-IV Axis I Disorders - Clinical Version (SCID-CV). Amercian Psychiatric Press.1997. [29] Slade M, Powell R, Rosen A, Strathdee G. Threshold Assessment Grid (TAG): the development of a valid and brief scale to assess the severity of mental illness. Soc Psychiatry Psychiatr Epidemiol 2000; 35: 78-85.

[30] Puschner B, Neumann P, Jordan H, Slade M, Fiorillo A, Giacco D, Egerhazi A, Ivanka T, Bording MK, Sorensen HO, Bär A, Kawohl W, Loos S. Development and psychometric 
properties of a five-language multiperspective instrument to assess clinical decision making style in the treatment of people with severe mental illness (CDMS). BMC Psychiatry 2013; 13: 48 doi:10.1186/1471-244X-13-48.

[31] Zeger SL, Liang KY, Albert PS. Models for longitudinal data: a generalized estimating equation approach. Biometrics 1988; 44: 1049-1060.

[32] Twisk JWR. Applied longitudinal data analysis for epidemiology: a practical guide.

Cambridge University Press. 2003.

[33] Coulter A, Magee H. The European patient of the future. Maidenhead, Open University Press, 2003.

[34] O’Donnell M, Monz B, Hunskaar S. General preferences for involvement in treatment decision-making among European women with urinary inconsistence. Soc Sci Med 2007; 64: 1914-1924.

[35] Andreassen HK, Bujnowska-Fedak MM, Chronaki CE, Dumitru RC, Pudule I, Santana S, Voss H, Wynn R. European citizens' use of E-health services: a study of seven countries. BMC Public Health, 2007; 7:53. doi:10.1186/1471-2458-7-53.

[36] Coulter A, Parsons S, Askham J. Policy brief: Where are the patients in decision-making about their own care? In WHO 2008 and WHO on behalf of the European Observatory on Health Systems and Policies 2008. Denmark: World Health Organisation; 2008.

[37] Mahone IH,. Shared decisison making and serious mental illness. Arch Psychiatry Nurs 2008 ; 22: 334.343. doi : 10.1016/j.apnu.2007.11.002.

[38] Patel SR, Bakken S. Preferences for participation in decision making among ethnically diverse patients with anxiety and depression. Community Ment Health J. 2010; 46: 466-473. doi: 10.1007/s10597-010-9323-3.

[39] Barry B, Henderson A. Nature of decision-making in the terminally ill patient. Cancer Nurs 1996; 19: 384-391.

[40] Caress AL, Luker K, Woodcok A, Beaver K. A qualitative exploration of treatment decision-making role preference in adult asthma patients. Health Expect 2002; 5: 223-235. 
[41] Lora, A. An overview of mental health system in Italy. Ann Ist Super Sanita 2009; 45: 516

[42] Neukirch, S. 30 Jahre Psychiatriereform in Italien - ein Rückblick auf den Reformprozess und seine sozial- und gesundheitspolitischen Einflussfaktoren. Sozialpsychiatrische Informationen 2008; 4: 2-11 DZI-SOLIT, ID: dzi-solit-0175528

[43] Doyal, L, Sheather, J. Mental health legislation should respect decision making capacity. BMJ 2005; 7530: 1467-1469.

[44] Degner LF, Sloan JA. Decision making during serious illness: What role do patients really want to play? J Clin Epidemiol 1992; 45: 941-950.

[45] Elwyn G, Edwards A. Evidence-based patient choice. Oxford: Oxford University Press. 2001.

[46] Leamy M, Bird V, Le Boutillier C, Williams J, Slade M. Conceptual framework for personal recovery in mental health: systematic review and narrative synthesis. $\mathrm{Br} \mathrm{J}$ Psychiatry 2001; 199: 445-452.

[47] Slade M, Leamy M, Bacon F, Janosik M, Le Boutillier C, Williams J, Bird V. International differences in understanding recovery: systematic review. Epidemiol Psychiatr Sci. 2012; 21:353-64. doi: 10.1017/S2045796012000133. 
Table 1: Baseline patient characteristics $(\mathrm{N}=514)$

\begin{tabular}{|c|c|c|c|c|c|c|c|c|}
\hline Patients & Total $(\mathrm{N}=514)$ & $\begin{array}{l}\text { Ulm } \\
(\mathrm{N}=96)\end{array}$ & $\begin{array}{l}\text { London } \\
(\mathrm{N}=69)\end{array}$ & $\begin{array}{l}\text { Naples } \\
(\mathrm{N}=92)\end{array}$ & $\begin{array}{l}\text { Debrecen } \\
(\mathrm{N}=88)\end{array}$ & $\begin{array}{l}\text { Aalborg } \\
(\mathrm{N}=81)\end{array}$ & $\begin{array}{l}\text { Zurich } \\
(\mathrm{N}=88)\end{array}$ & $\begin{array}{l}\text { Center } \\
\text { differences }\end{array}$ \\
\hline \multicolumn{9}{|l|}{ Gender } \\
\hline $\begin{array}{l}\text { Female, n (\%) } \\
\text { Age }\end{array}$ & $269(52.30)$ & $62(64.60)$ & $20(29.00)$ & $47(51.10)$ & $44(50.00)$ & $40(49.40)$ & $56(63.60)$ & $p<0.001$ \\
\hline Years, M (SD) & $41.61(10.75)$ & $45.29(8.82)$ & $37.05(10.30)$ & $41.93(11.19)$ & $45.17(9.81)$ & $39.76(10.99)$ & $38.97(10.96)$ & $\mathrm{p}<0.001$ \\
\hline \multicolumn{9}{|l|}{ Marital Status } \\
\hline $\begin{array}{l}\text { Married, n (\%) } \\
\text { Ethnic group }\end{array}$ & \multicolumn{7}{|c|}{ Ethnic group } & $p<0.001$ \\
\hline $\begin{array}{l}\text { White, } n(\%) \\
\text { Years in school }\end{array}$ & $487(94.90)$ & $96(100.00)$ & $45(65.20)$ & $92(100.00)$ & $88(100.00)$ & $80(98.8)$ & 86 (98.90) & $p<0.001$ \\
\hline \multicolumn{9}{|l|}{ Living situation } \\
\hline $\begin{array}{l}\text { Alone, n (\%) } \\
\text { Parents, n (\%) } \\
\text { Other, n (\%) }\end{array}$ & $\begin{array}{l}195(38.20) \\
103(20.20) \\
213(41.60)\end{array}$ & $\begin{array}{l}53(55.20) \\
7(7.30) \\
36(37.50)\end{array}$ & $\begin{array}{l}17(24.60) \\
10(14.50) \\
42(60.90)\end{array}$ & $\begin{array}{l}10(11.00) \\
40(44.00) \\
41(45.00)\end{array}$ & $\begin{array}{l}20(22.70) \\
33(37.50) \\
35(39.80)\end{array}$ & $\begin{array}{l}49(60.50) \\
7(8.60) \\
25(30.90)\end{array}$ & $\begin{array}{l}46(53.50) \\
6(7.00) \\
34(39.50)\end{array}$ & $p<0.001$ \\
\hline \multicolumn{9}{|l|}{ Employment Status } \\
\hline $\begin{array}{l}\text { Employed, n (\%) } \\
\text { State benefits }\end{array}$ & $97(18.90)$ & $25(26.00)$ & $1(1.4)$ & $25(27.20)$ & $15(17.00)$ & $6(7.40)$ & $25(28.40)$ & $\mathrm{p}<0.001$ \\
\hline $\begin{array}{l}\text { Yes, n (\%) } \\
\text { Diagnosis }\end{array}$ & $372(72.40)$ & $78(81.20)$ & $58(84.10)$ & $21(22.80)$ & $77(87.50)$ & $76(93.80)$ & $62(70.50)$ & $\mathrm{p}<0.001$ \\
\hline $\begin{array}{l}\text { Psychotic disorder, n (\%) } \\
\text { Mood disorder, n (\%) } \\
\text { Other, n (\%) }\end{array}$ & $\begin{array}{l}236(45.90) \\
173(33.70) \\
105(20.30)\end{array}$ & $\begin{array}{l}34(35.40) \\
43(44.80) \\
19(19.80)\end{array}$ & $\begin{array}{l}49(71.00) \\
14(20.30) \\
6(8.70)\end{array}$ & $\begin{array}{l}22(23.90) \\
39(42.40) \\
31(33.70)\end{array}$ & $\begin{array}{l}50(56.80) \\
19(21.60) \\
19(21.60)\end{array}$ & $\begin{array}{l}53(65.40) \\
19(23.50) \\
9(11.10)\end{array}$ & $\begin{array}{l}28(31.80) \\
39(44.30) \\
21(23.90)\end{array}$ & $\mathrm{p}<0.001$ \\
\hline
\end{tabular}


Table 2: Repeated measurement of the $\mathrm{CDMS}^{1}$ in association with center location

\begin{tabular}{|c|c|c|c|c|c|}
\hline & & \multicolumn{3}{|l|}{ Means (SE) } & \multirow[t]{2}{*}{ Pairwise comparisons } \\
\hline & & T0 & T6 & Pooled & \\
\hline \multirow[t]{13}{*}{ Center } & Zurich, Switzerland & $2.04(0.06)$ & $2.27(0.04)$ & $2.15(0.04)$ & Zurich, London, Ulm > Debrecen > Naples \\
\hline & Aalborg, Denmark & $1.97(0.06)$ & $1.99(0.06)$ & $1.98(0.05)$ & London $>$ Aalborg $>$ Debrecen $>$ Naples \\
\hline & Debrecen, Hungary & $1.52(0.04)$ & $1.55(0.05)$ & $1.53(0.04)$ & \\
\hline & Naples, Italy, & $1.20(0.06)$ & $1.05(0.04)$ & $1.12(0.04)$ & \\
\hline & London, England & $2.26(0.07)$ & $2.31(0.08)$ & $2.28(0.07)$ & \\
\hline & Ulm, Germany & $2.10(0.05)$ & $2.18(0.06)$ & $2.14(0.05)$ & \\
\hline & & b & $95 \% \mathrm{Cl}$ & Sig. & \\
\hline & Zurich * Follow-up & 0.230 & $0.147 ; 0.312$ & 0.000 & \\
\hline & Aalborg * Follow-up & 0.022 & $-0.066 ; 0.111$ & 0.625 & \\
\hline & Debrecen * Follow-up & 0.034 & $-0.064 ; 0.133$ & 0.492 & \\
\hline & Naples * Follow-up & -0.150 & $-0.277 ;-0.024$ & 0.020 & \\
\hline & London * Follow-up & 0.055 & $-0.107 ; 0.217$ & 0.506 & \\
\hline & Ulm * Follow-up & 0.085 & $-0.010 ; 0.180$ & 0.079 & \\
\hline
\end{tabular}

${ }^{1}$ CDMS: Clinical Decision Making Style 
Table 3: Repeated measurement of the CDMS ${ }^{1}$ in association with center location, adjusted for patient's age, marital status, living situation, state benefits, as well as repeated TAG scores

\begin{tabular}{|c|c|c|c|c|c|}
\hline & & \multicolumn{3}{|l|}{ Means (SE) } & \multirow[t]{2}{*}{ Pairwise comparisons } \\
\hline & & T0 & T6 & Pooled & \\
\hline \multirow{13}{*}{ Center } & Zurich, Switzerland & $2.03(0.06)$ & $2.26(0.05)$ & $2.14(0.05)$ & Zurich, London, Ulm > Debrecen > Naples \\
\hline & Aalborg, Denmark & $1.95(0.07)$ & $1.99(0.07)$ & $1.97(0.06)$ & London $>$ Aalborg $>$ Debrecen $>$ Naples \\
\hline & Debrecen, Hungary & $1.54(0.05)$ & $1.58(0.05)$ & $1.56(0.05)$ & \\
\hline & Naples, Italy, & $1.21(0.06)$ & $1.07(0.05)$ & $1.14(0.04)$ & \\
\hline & London, England & $2.23(0.08)$ & $2.31(0.10)$ & $2.27(0.08)$ & \\
\hline & Ulm, Germany & $2.10(0.05)$ & $2.17(0.07)$ & $2.13(0.05)$ & \\
\hline & & b & $95 \% \mathrm{Cl}$ & Sig. & \\
\hline & Zurich * Follow-up & 0.233 & $0.132 ; 0.335$ & 0.000 & \\
\hline & Aalborg * Follow-up & 0.042 & $-0.055 ; 0.140$ & 0.391 & \\
\hline & Debrecen * Follow-up & 0.037 & $-0.062 ; 0.135$ & 0.464 & \\
\hline & Naples * Follow-up & -0.143 & $-0.272 ;-0.014$ & 0.030 & \\
\hline & London * Follow-up & 0.075 & $-0.100 ; 0.250$ & 0.401 & \\
\hline & Ulm * Follow-up & 0.074 & $-0.031 ; 0.179$ & 0.165 & \\
\hline
\end{tabular}

${ }^{1}$ CDMS: Clinical Decision Making Style 
